\title{
A rare case of malignant solitary fibrous tumor in prostate with review of the literature
}

\author{
Andrea Ronchi', Elvira La Mantia', Vincenzo Gigantino ${ }^{2}$, Sisto Perdonà ${ }^{3}$, Marco De Sio ${ }^{4}$, Gaetano Facchini ${ }^{5}$, \\ Renato Franco ${ }^{1 *}$ (D) and Annarosaria De Chiara $^{2}$
}

\begin{abstract}
Background: Solitary fibrous tumor is an uncommon soft tissue neoplasm with intermediate biological behavior, which rarely metastasizes. Malignant solitary fibrous tumor, although not clearly defined, is rarely described in the prostate. The present case is characterized by some peculiarities if compared with previously reported cases of prostatic malignant solitary fibrous tumor. Firstly, it does not show a homogeneous morphology: part of the neoplasm (about 50\%) showed the features of a conventional solitary fibrous tumor, while the remaining part showed the features of a malignant solitary fibrous tumor. In addition, the case is the first malignant solitary fibrous tumor reaching a huge diameter of $20 \mathrm{~cm}$ and replacing all prostatic parenchyma. Interestingly, normal prostatic parenchyma was observed on left-lobe trans-rectal needle-core biopsies, but was totally absent in surgical specimen. Since radical prostatectomy was carried out about 4 months after the biopsies, such discordant data may suggest exceedingly rapid growth of the neoplasm.

Case presentation: We report a case of a 62-year-old male, presented at medical observation for urinary retention, constipation and an enlarged prostate gland. A trans-rectal prostatic biopsy showed a low-grade spindle cell neoplasm. Histopathological examination of the prostatectomy specimen showed patternless architecture with hypocellular and hypercellular areas and hemangiopericytoma-like vessels. In some fields the neoplasm was characterized by a high mitotic index and evident cellular atypia. Immunohistochemically, neoplastic cells were positive for CD34, bcl2, CD99, STAT6 and partially for PgR. The neoplasm was diagnosed as a malignant solitary fibrous tumor.

Conclusions: The differential diagnosis of spindle cells tumors arising in the prostrate is broad and includes lesions of epithelial and mesenchymal origin, primary prostatic lesions such as stromal tumors of uncertain malignant potential and stromal sarcoma, as well as anatomically ubiquitous soft tissue neoplasms. Solitary fibrous tumors should be considered in cases of prostatic tumors with a spindled morphology, but malignancy in such tumors is extremely rare in the prostate. A review of literature showed only four additional cases. Because of the unpredictable biological behavior and the possibility of recurrence, a long-term clinical and instrumental follow-up is recommended.
\end{abstract}

Keywords: Mesenchymal neoplasm, Prostate neoplasm, Spindle cell tumor, Haemangiopericytoma, Soft tissue tumor

\footnotetext{
* Correspondence: renato.franco@unicampania.it

'Pathology Unit, Università della Campania "L. Vanvitelli", Via Luciano

Armanni, 80138 Naples, Italy

Full list of author information is available at the end of the article
} 


\section{Background}

Solitary fibrous tumor (SFT) is a rare mesenchymal neoplasm of postulated myofibroblastic origin [1]. The definitive etiology of SFT remains unknown, but some pathogenic mechanisms have been described. In fact, the tumor is often associated with NAB2-STAT6 gene fusion, arising from recurrent intra-chromosomal rearrangements on chromosome 12q13 [2]. This genetic alteration deregulates the expression of $N A B 2$, an important regulator of the transforming growth factor $\beta$ (TGF $\beta$ ), and STAT6 (signal transducer and activator of transcription), a transcriptional factor modulating signaling through interleukin-4 and interleukin-13. The genetic fusion can occur at different breakpoints and distinct fusion types may be associated with distinct clinic-pathologic subgroups of SFT [3]. The phenotypical effect of this rearrangement is nuclear expression of the C-terminal portion of STAT6 [4]. SFT is labeled as an intermediate, rarely metastasizing, neoplasm with variable clinical behavior [1]. A more aggressive variant is malignant SFT, with higher rates of local recurrences and distant metastasis [5]. Despite wide surgical excision, estimated recurrence rate for malignant SFT is approximately 30\% [5]. A recently described entity is dedifferentiated SFT, which shows abrupt transition from conventional SFT to a high-grade sarcoma [6]. Wide surgical excision is the most important prognostic factor and prognosis is worse in patients with metastatic disease, with median survival ranging from 22 to 46 months [5]. For unresectable disease, chemotherapy and radiation therapy showed variable efficacy [7].

\section{Case presentation}

A 62-years-old man was admitted to the National Cancer Institute of Naples with urinary retention and lower urinary tract symptoms. Serum prostate-specific antigen (PSA) level was $5.80 \mathrm{ng} / \mathrm{ml}$. The prostate was enlarged, smooth and firm on digital rectal examination, and a trans-rectal needle core biopsy was planned. Histologically, the tissue from the left lobe showed normal prostatic parenchyma, while cores rom the right lobe were completely occupied by a patternless neoplastic proliferation of ovoid and spindle cells with ill-defined cellular borders and relatively homogeneous nuclei, embedded in a variable quantity of fibrous stroma. Irregularly shaped, "hemangiopericytoma-like" vessels were evident, while neither necrotic areas nor mitosis were present. Immunohistochemical examination revealed that the neoplastic cells were positive for CD34 and bcl2, partially positive for progesterone receptor (PgR) and negative for cytokeratin (CK), PSA, smooth muscle actin (SMA), calponin and CD117. A diagnosis of low grade sarcoma was made, deferring the final diagnosis to the surgical specimen. Computed Tomography (CT) of the pelvis revealed a huge lobulated mass of about $20 \times 10 \mathrm{~cm}$ involving the prostate gland and pushing the bladder base. The patient underwent radical prostatectomy about 4 months after the biopsy. Gross evaluation of the surgical specimen revealed a well-defined, whitish and lobulated mass with a central area of necrosis (Fig. 1). Histologically, the neoplasm presented variable appearance with alternating hypocellular and hypercellular areas. Hypocellular areas showed a patternless population of ovoid cells and a large number of irregular blood vessels. The central area of the neoplasia was hypercellular with spindle cells forming short compact fascicles arranged in a whirling pattern, moderately atypical nuclei and some evident nucleoli. In this area, about 8 mitosis per 10 high power fields and the presence of necrosis were evident. Interestingly, no residual prostatic parenchyma was found. The tumor was generally well demarcated, but showed infiltrating behavior close to the right seminal vesicle; the margins of surgical resection, however, were free of neoplasia. Histological features of the neoplasm are summarized in Fig. 2. Immunohistochemically, the tumor was positive for vimentin, CD34, CD99

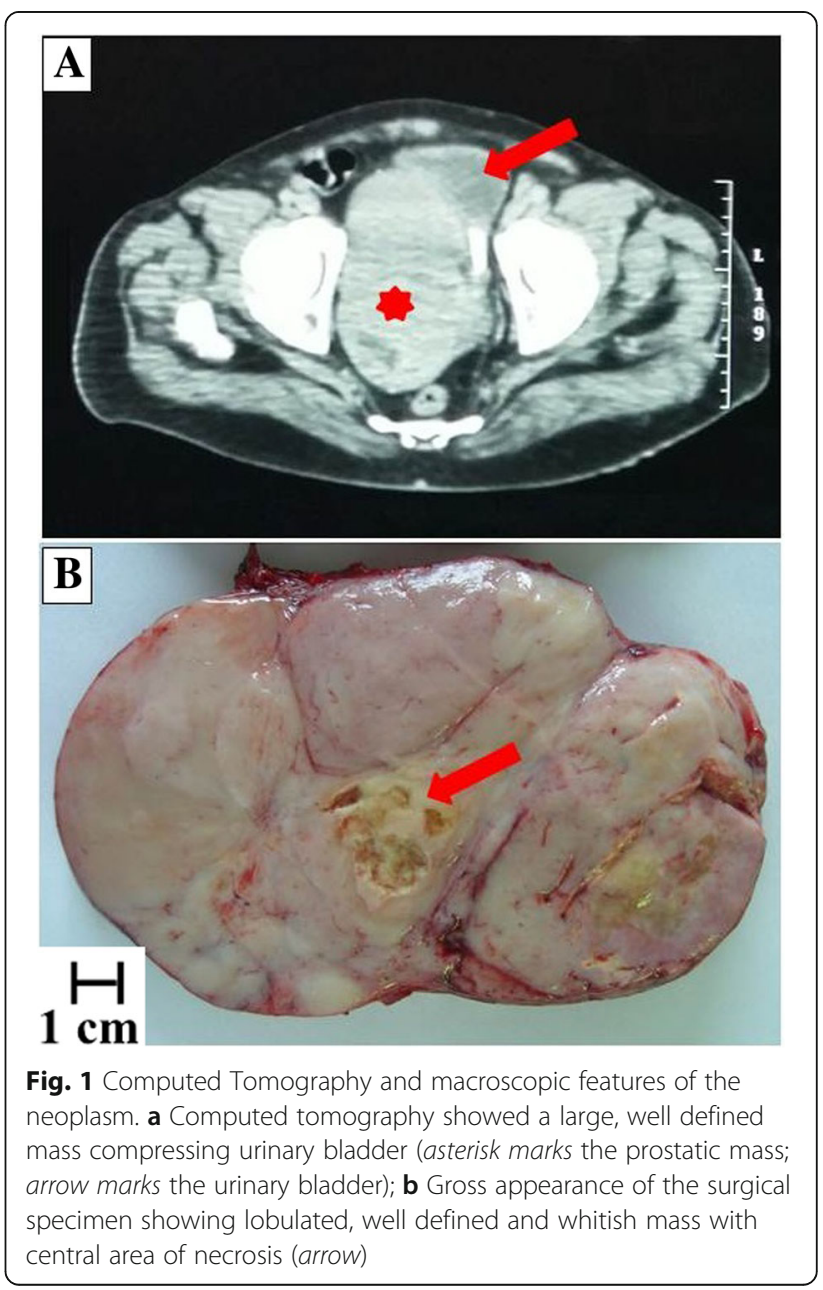




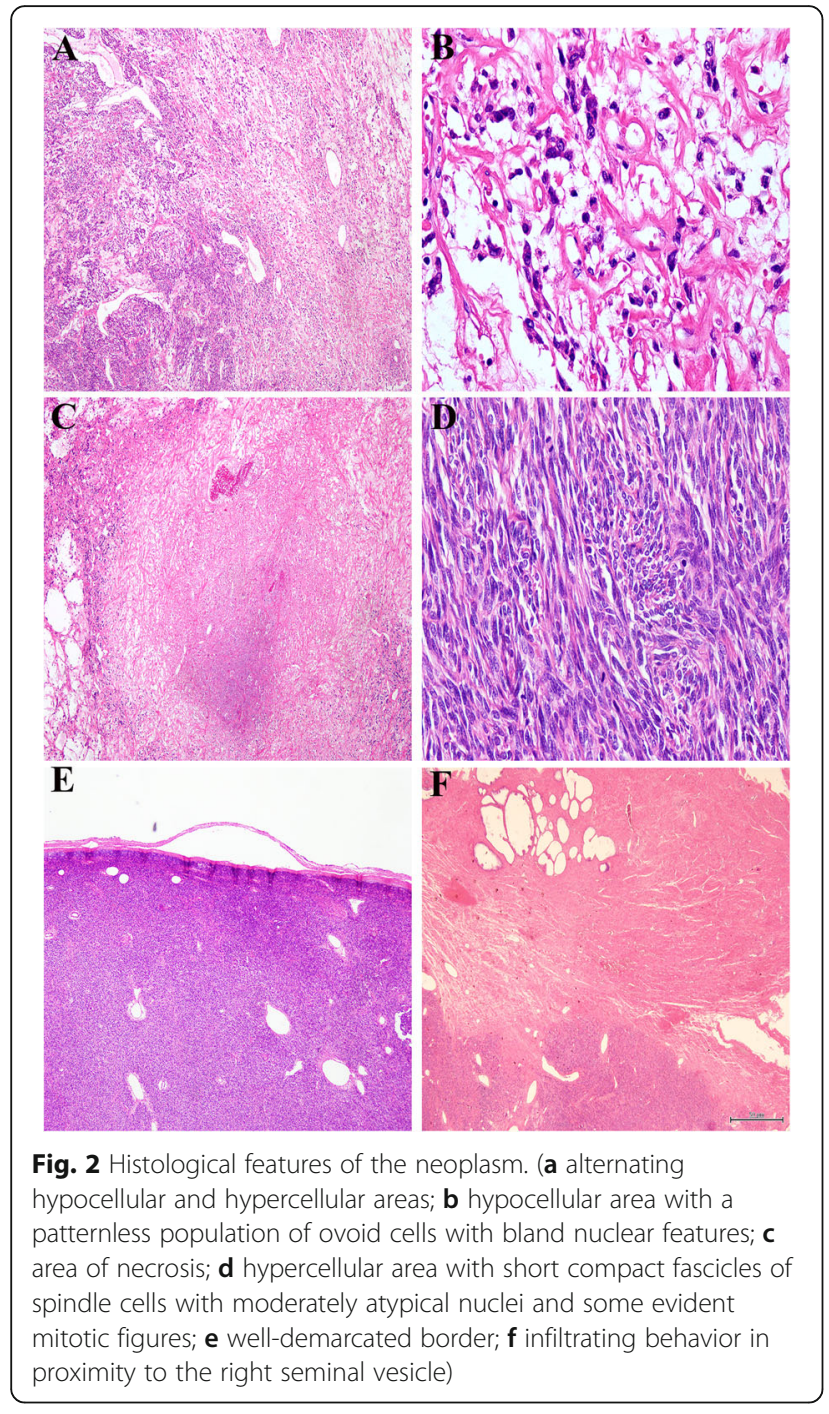

and bcl2 while it was immunonegative for CK, SMA, S100, Epithelial Membrane Protein (EMA), desmin, calponin, CD10, CD117, estrogen receptor (ER), B-catenin. About $40 \%$ of neoplastic cells stained for progesterone receptor (PgR). A final diagnosis of malignant solitary fibrous tumor was made. Some years later, in the course of the re-examination of a series of SFTs from our files, the diffuse STAT6 nuclear positivity in the present case further confirmed the diagnosis. Immunohistochemical features of the neoplasm are summarized in Fig. 3. No adjuvant therapy was administered after surgery and no recurrences were recorded in a follow up 8 years later.

\section{Discussion}

The onset of a malignant SFT is an exceedingly rare event, especially in the prostate, and diagnosis can be challenging, as most of the prostatic mesenchymal neoplasms are characterized by spindle cell morphology with frequent overlapping in histological features.
Differential diagnosis includes a broad variety of lesions, both histotypes restricted to the prostate and anatomically ubiquitous soft tissue neoplasms [8]. It is particularly difficult to differentiate prostatic malignant SFT from prostatic stromal sarcoma (PSS) and gastrointestinal stromal tumors (GIST). PSS is a spindle cell neoplasm, which may have storiform, epithelioid or fibrosarcomatous pattern. The neoplasm is characterized by stromal overgrowth with hypercellularity and a variable degree of necrosis, cytologic atypia and mitotic activity [9]. The stromal component can be solid or mixed with benign glandular elements. Primary prostatic GIST has been rarely reported in literature, and more frequently the neoplasm arises from the rectum or perirectal space with subsequent involvement of the prostate $[10,11]$. Histologically, GIST shows spindle cells with bland nuclei and perinuclear vacuoles, organized in a fascicular growth pattern. A correct pre-operative diagnosis of GIST involving the prostate is particularly important because of the possibility of medical treatment with tyrosine kinase inhibitors, compared with the surgical treatment of most other prostatic mesenchymal lesions [12]. The appropriate use of immunohistochemistry is always necessary for an accurate diagnosis. In this setting, the most helpful markers are SMA, desmin, CK, CD117, STAT6, CD34, PgR, bcl 2 and CD99. Immunohistochemical stainings in prostatic mesenchymal lesions is summarized in Table 1. PgR is frequently expressed in PSS, supporting its derivation from the hormonally responsive prostate mesenchyme [13, 14]. However PgR expression has been variably described in other prostatic mesenchymal neoplasms including smooth muscle tumours and SFT $[15,16]$. Diagnosis of SFT has classically been based on the immunohistochemical expression of markers such as CD34, bcl2 and CD99. Nevertheless, recent studies have demonstrated the poor specificity of these markers [17]. CD34 is expressed significantly in a large number of soft tissue neoplasms and many entities that are included in the differential diagnosis of SFT, such as PSS and GIST, share CD34 expression [11]. Furthermore CD34 expression is absent in approximately $5-10 \%$ of conventional SFT and in the large majority of malignant and dedifferentiated forms $[18,19]$. Therefore the absence of $\mathrm{CD} 34$ expression does not exclude the diagnosis of SFT. The expression of Bcl2 and CD99 are variably used to support the diagnosis of SFT but are less sensitive than CD34 and equally non-specific [17]. Recently, the discovery of the NAB2-STAT6 fusion gene in SFT led to development of a STAT6 antibody that is a reliable immunohistochemical marker with a high level of sensitivity and specificity [4-20]. Therefore, nuclear expression of STAT6 is currently the most useful marker for distinguishing SFT from its histologic mimics. STAT6 nuclear expression has been demonstrated both 


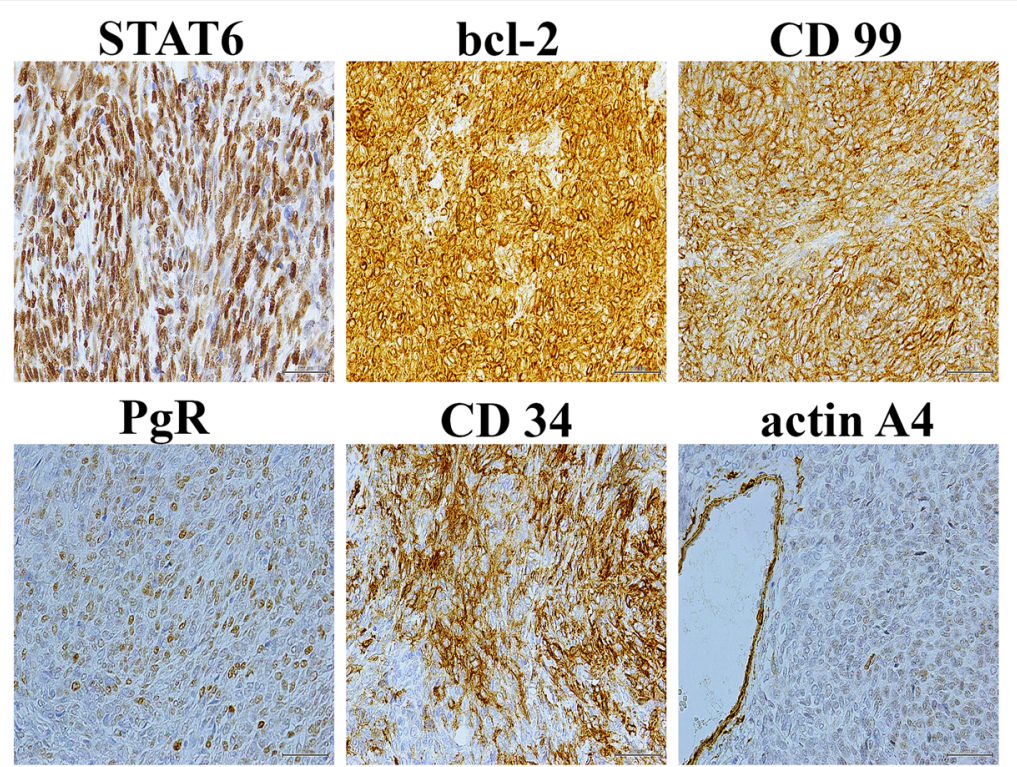

Fig. 3 Immunohistochemical features of the neoplasm. Neoplastic cells are diffusely positive for STAT6, bcl-2, CD99 and CD34; partially positive for PgR and negative for actin A4

in conventional and in malignant SFT, while dedifferentiated forms frequently lose STAT6 expression [21]. Remarkably, the presence of the chimeric fusion gene NAB2-STAT6 is not demonstrable by in-situ hybridization (ISH) methods, due to the natural proximity of the two genes [22]. Genetic studies have recently established distinct NAB2-STAT6 fusion gene variants associated with different clinical behavior. In particular, NAB2 exon 6 - STAT6 exon 16/17/18 variants seem to be associated with malignant morphology and aggressive clinical course, while NAB2 exon 4 -STAT6 exon $2 / 3$ variants are associated with pleural origin and less aggressive clinico-pathological features [23]. Recently, immunohistochemical expression of ALDH1 (Aldehyde Dehydrogenase 1), an intracytoplasmatic enzyme highly expressed in stem cells, has been demonstrated in SFT $[24,25]$. In one study, cytoplasmic ALDH1 expression was shown to be positive in $76 \%$ of SFTs [25]. Thus, the use of both STAT6 and ALDH1 immunostaining increases significantly the specificity of SFT diagnosis especially in the differential diagnosis with stromal tumor of uncertain malignant potential (STUMP) and SS [24]. SFT is classified in the 2013 edition of the World Health Organization (WHO) classification of tumors of soft tissue as a fibroblastic/myofibroblastic neoplasm with intermediate-rarely metastasizing biological behavior [1]. However the clinical behavior of individual tumors is not easily predictable. A clinicopathological study of 110 SFT cases by Demicco et al. elaborated a risk stratification model including age, tumor dimension and mitotic figures. Thus, SFT patients with higher risk of progression were characterized by tumor size larger than $15 \mathrm{~cm}$, age higher than 55 years and mitotic figures more than 4/10 highpower fields [26]. According to this model, our patient had the highest risk score; nevertheless, no

Table 1 Immunohistochemical features of spindle cell lesions of the prostate

\begin{tabular}{llllllllll}
\hline & pankeratin & SMA & Desmin & Myogenin & CD34 & PSA & PgR & CD117 & STAT6 \\
\hline STUMP & neg & neg/pos & neg/pos & neg & pos & neg & pos & neg & neg \\
PSS & neg & neg/pos & neg/pos & neg & pos & neg & pos & neg & neg \\
SMT & neg/pos & pos & pos & neg & neg & neg & pos $/$ neg & neg & neg \\
IMT & neg/pos & pos & pos & neg & neg & neg & neg & pos $/$ neg & neg \\
SFT & neg & neg/pos & neg & neg & pos & neg & pos $/$ neg & neg & pos \\
GIST & neg & pos/neg & pos/neg & neg & pos & neg & neg & pos & neg \\
SC & pos/neg & neg/pos & neg/pos & neg & neg & pos $/$ neg & neg & neg & neg \\
\hline
\end{tabular}

STUMP stromal tumor or uncertain malignant potential, PSS prostatic stromal sarcoma, SMT smooth muscle tumors, IMT inflammatory myofibroblastic tumor, SFT solitary fibrous tumor, GIST gastrointestinal stromal tumor, SC sarcomatoid carcinoma, RMS rhabdomyosarcoma, SMA smooth muscle actin, CD34 cluster of differentiation 34, PSA prostate-specific antigen, PgR progesterone receptor, CD117 cluster of differentiation 117, STAT6 signal transduction and activation of transcription 6 
recurrence or metastases were found in a follow up 8 years later.

While several cases of conventional SFT have been reported in the prostate, the onset of malignant SFT in the prostate is an extremely rare event and the review of the literature reveals only four other cases. In 2005 Vodovnik et al. described a case of malignant SFT in a 87 years-old man [27]. The patient presented massive haematuria and died on the first post-operative day following a cardiac arrest. Surgical specimen consisted of five nodular pieces of prostatic parenchyma, measuring $2-9 \mathrm{~cm}$ in greatest diameter. Three other cases were further described by Herawi and Epstein in 2007 [28]. These cases were diagnosed on either needle biopsies or transurethral resections of the prostate. Follow-up was available for two patients showing that both patients were alive and without any evidence of disease at 5 and 10 years after the initial diagnosis. Unfortunately other clinical details were not described by the authors. The case we present is also characterized by a particular clinical behavior. This is the first described case of malignant SFT, which reaches a huge diameter of $20 \mathrm{~cm}$ and replaces the whole prostatic parenchyma. Interestingly, normal prostatic parenchyma was present on left-lobe trans-rectal needle-core biopsies, but it was totally absent in surgical specimen. Since radical prostatectomy was performed about 4 months after the biopsies, this peculiarity may suggest an exceeding rapid growth of the neoplasm.

\section{Conclusions}

SFT is a rare neoplasm of mesenchymal origin that should be considered in cases of prostatic tumor with a spindled morphology. Positive nuclear staining for STAT6 is currently the most accurate diagnostic test. Considering the low predictable biological behavior and the possibility of recurrence and metastases, a long-term clinical and instrumental follow-up is recommended.

\section{Abbreviations \\ ALDH1: Aldehyde dehydrogenase 1; bcl2: b-cell lymphoma 2; CD: Cluster of differentiation; CK: Cytokeratin; CT: Computed tomography; EMA: Epithelial membrane protein; ER: Estrogen receptor; GIST: Gastrointestinal stromal tumor; IMT: Inflammatory myofibroblastic tumor; ISH: in-situ hybridization; ml: milliliters; ng: nanograms; PgR: Progesterone receptor; PSA: Prostate- specific antigen; PSS: Prostatic stromal sarcoma; RMS: Rhabdomyosarcoma; SC: Sarcomatoid carcinoma; SFT: Solitary fibrous tumor; SMA: Smooth muscle actin; SMT: Smooth muscle tumors; STAT6: Signal transducer and activator of transcription 6; STUMP: Stromal tumor of uncertain malignant potential; TGF $\beta$ : Transforming growth factor $\beta$; WHO: World Health Organization}

\section{Acknowledgements}

The authors thank Tom Nevin for editing the language of this paper.

\section{Funding}

No funding was received for this case report.

Availability of data and materials Not applicable.

\section{Authors' contributions}

$\mathrm{RF}$ and $\mathrm{AD}$ conceived and designed the work. AR and EL wrote the manuscript. SP, MD, GF acquired the samples and the data. VG performed the experiments. AR, RF and AD discussed results and performed the review of literature. RF supervised the work. All Authors read and approved the final manuscript.

Ethics approval and consent to participate

Not applicable.

\section{Consent for publication}

Written informed consent was obtained from the patient for publication of this case report and any accompanying images. A copy of the written consent is available for review by the Editor-in-Chief of this journal.

\section{Competing interests}

The authors declare that they have no competing interests.

\section{Publisher's Note}

Springer Nature remains neutral with regard to jurisdictional claims in published maps and institutional affiliations.

\section{Author details}

'Pathology Unit, Università della Campania "L. Vanvitelli", Via Luciano Armanni, 80138 Naples, Italy. ${ }^{2}$ Pathology Unit, Istituto Nazionale Tumori I. R. C. C. S. "Fondazione Pascale", Naples, Italy. ${ }^{3}$ Division of Urology, Department of Uro-Gynaecological Oncology, Istituto Nazionale Tumori I. R. C. C. S. "Fondazione Pascale", Naples, Italy. ${ }^{4}$ Division of Urology, Università della Campania "L. Vanvitelli", Naples, Italy. ${ }^{5}$ Division of Medical Oncology, Department of Uro-Gynaecological Oncology, Istituto Nazionale Tumori I. R. C. C. S. "Fondazione Pascale", Naples, Italy.

Received: 23 February 2017 Accepted: 29 June 2017 Published online: 07 July 2017

\section{References}

1. Fletcher CDM, Bridge JA, Lee JC. Extrapleural solitary fibrous tumour. In: Fletcher CDM, editor. WHO classification of tumours ofsoft tissue and bone. 4th ed. Lyon: the International Agency for Research on Cancer press; 2013. p. $80-2$.

2. Robinson DR, Wu YM, Kalyana-Sundaram S, et al. Identification of recurrent NAB2-STAT6 gene fusions in solitary fibrous tumor by integrative sequencing. Nat Genet. 2013;45:180-5.

3. Barthelmess S, Geddert H, Boltze C, et al. Solitary fibrous tumors/ hemangiopericytomas with different variants of the NAB2-STAT6 gene fusion are characterized by specific histomorphology and distinct clinicopathological features. Am J Pathol. 2014;184:1209-18.

4. Doyle LA, Vivero M, Fletcher CD, Mertens F, Hornick JL. Nuclear expression of STAT6 distinguishes solitary fibrous tumor from histologic mimics. Mod Pathol. 2014:27(3):390-5.

5. Lococo F, Cesario A, Cardillo G, et al. Malignant solitary fibrous tumors of the pleura: retrospective review of a multicenter series. J Thorac Oncol. 2012;7:1698-706.

6. Mosquera JM, Fletcher CD. Expanding the spectrum of malignant progression in solitary fibrous tumors: a study of 8 cases with a discrete anaplastic component-is this dedifferentiated SFT? Am J Surg Pathol. 2009; 33(9):1314-21.

7. Khalifa J, Ouali M, Chaltiel L, et al. Efficacy of trabectedin in malignant solitary fibrous tumors: a retrospective analysis from the French sarcoma group. BMC Cancer. 2015;15:700.

8. Paner GP, Aron M, Hansel DE, Amin MB. Non-epithelial neoplasms of the prostate. Histopathology. 2012;60:166-86.

9. Cheville J, Algaba F, Epstein Jl, Lopez-Beltran A. In: Moch H, editor. WHO classification of tumours of the urinary system and male genital organs. 4th ed. Lyon: the International Agency for Research on Cancer press; 2016. p. 177.

10. Herawi M, Montgomery EA, Epstein J. Gastrointestinal stromal tumors (GISTs) on prostate needle biopsy: a clinicopathologic study of 8 cases. Am J Surg Pathol. 2006;30:1389-95.

11. Hansel DE, Netto GJ, Montgomery EA, Epstein JI. Mesenchymal tumors of the prostate. Surg Pathol Clin. 2008;1(1):105-28. 
12. Arce-Lara C, Shah MH, Jimenez RE, et al. Gastrointestinal stromal tumors involving the prostate: presentation, course, and therapeutic approach. Urology. 2007;69:1209.

13. Gaudin PB, Rosai J, Epstein Jl. Sarcomas and related proliferative lesions of specialized prostatic stroma: a clinicopathologic study of 22 cases. Am J Surg Pathol. 1998;22:148-62.

14. Hossain D, Meiers I, Qian J, Mac Lennan GT, Bostwick DG. Prostatic stromal hyperplasia with atypia: follow-up study of 18 cases. Arch Pathol Lab Med. 2008;132:1729-33.

15. Hossain D, Meiers I, Qian J, Mac Lennan GT, Bostwick DG. Prostatic leiomyoma with atypia: follow-up study of 10 cases. Ann Diagn Pathol. 2008;12:328-32.

16. Paner GP, Aron M, Hansel DE, et al. Non-epithelial neoplasms of the prostate. Histopathology. 2012;60(1):166-86.

17. Miettinen M. Immunohistochemistry of soft tissue tumours - review with emphasis on 10 markers. Histopathology. 2014;64(1):101-18.

18. Masuda Y, Kurisaki-Arakawa A, Hara K, et al. A case of dedifferentiated solitary fibrous tumor of the thoracic cavity. Int J Clin Exp Pathol. 2014;7:386-93.

19. Kurisaki-Arakawa A, Akaike $K$, Hara $K$, et al. A case of dedifferentiated solitary fibrous tumor in the pelvis with p53 mutation. Virchows Arch. 2014:465:615-21.

20. Koelsche C, Schweizer L, Renner M, et al. Nuclear relocation of STAT6 reliably predicts NAB2/STAT6 fusion for the diagnosis of solitary fibrous tumour. Histopathology. 2014;65:613-22.

21. Doyle LA, Tao D, Mariño-Enríquez A. STAT6 is amplified in a subset of dedifferentiated liposarcoma. Mod Pathol. 2014;27(9):1231-7.

22. Akaike K, Kurisaki-Arakawa A, Hara K, et al. Distinct clinicopathological features of NAB2-STAT6 fusion gene variants in solitary fibrous tumor with emphasis on the acquisition of highly malignant potential. Hum Pathol. 2015:46:347-56

23. Barthelmeß S, Geddert H, Boltze C, et al. Solitary fibrous tumors/ hemangiopericytomas with different variants of the NAB2-STAT6 gene fusion are characterized by specific histomorphology and distinct clinicopathological features. Am J Pathol. 2014;184:1209-18.

24. Guner G, Bishop JA, Bezerra SM, et al. The utility of STAT6 and ALDH1 expression in the differential diagnosis of solitary fibrous tumor versus prostate-specific stromal neoplasms. Hum Pathol. 2016;54:184-8.

25. Ouladan S, Trautmann M, Orouji E, et al. Differential diagnosis of solitary fibrous tumors: a study of 454 soft tissue tumors indicating the diagnostic value of nuclear STAT6 relocation and ALDH1expression combined with in situ proximity ligation assay. Int J Oncol. 2015;46(6):2595-605.

26. Demicco EG, Park MS, Araujo DM, et al. Solitary fibrous tumor: a clinicopathologicalstudy of 110 cases and proposed risk assessment model. Mod Pathol. 2012;25(9):1298-306.

27. Vodovnik A, Rogawski K, Bolton JF. A case of malignant solitary fibrous tumor of the prostate. Pathol Int. 2005;55(12):807-8.

28. Herawi M, Epstein Jl. Solitary fibrous tumor on needle biopsy and transurethral resection of the prostate: a clinicopathologic study of 13 cases. Am J Surg Pathol. 2007;31(6):870-6.

\section{Submit your next manuscript to BioMed Central and we will help you at every step:}

- We accept pre-submission inquiries

- Our selector tool helps you to find the most relevant journal

- We provide round the clock customer support

- Convenient online submission

- Thorough peer review

- Inclusion in PubMed and all major indexing services

- Maximum visibility for your research

Submit your manuscript at www.biomedcentral.com/submit

) Biomed Central 